\title{
The connection between W31, SGR 1806-20, and LBV 1806-20: Distance, extinction, and structure
}

\author{
S. Corbel ${ }^{1}$ and S. S. Eikenberry ${ }^{2, \star}$ \\ 1 Université Paris VII and Service d'Astrophysique, CEA Saclay, 91191 Gif-sur-Yvette Cedex, France \\ 2 Department of Astronomy, University of Florida, 211 Bryant Space Science Center, Gainesville, FL 32611, USA \\ e-mail: eiken@astro.ufl.edu
}

Received 4 July 2003 / Accepted 3 October 2003

\begin{abstract}
We present new millimeter and infrared spectroscopic observations towards the radio nebula G10.0-0.3, which is powered by the wind of the Luminous Blue Variable star LBV 1806-20 also closely associated with the soft gamma-ray repeater SGR 1806-20, and believed to be located in the giant Galactic H II complex W31. Based on observations of CO emission lines and $\mathrm{NH}_{3}$ absorption features from molecular clouds along the line of sight to G10.0-0.3, as well as the radial velocity and optical extinction of the star powering the nebula, we determine its distance to be $15.1_{-1.3}^{+1.8} \mathrm{kpc}$ in agreement with Corbel et al. (1997). In addition, this strengthens the association of SGR 1806-20 with a massive molecular cloud at the same distance. All soft gamma-ray repeaters with precise location are now found to be associated with a site of massive star formation or molecular cloud. We also show that W31 consists of at least two distinct components along the line of sight. We suggest that G10.2-0.3 and G10.6-0.4 are located on the $-30 \mathrm{~km} \mathrm{~s}^{-1}$ spiral arm at a distance from the Sun of $4.5 \pm 0.6 \mathrm{kpc}$ and that G10.3-0.1 may be associated with a massive molecular cloud at the same distance as the LBV star, i.e. $15.1_{-1.3}^{+1.8} \mathrm{kpc}$, implying that W31 could be decomposed into two components along the line of sight.
\end{abstract}

Key words. stars: neutron - stars: individual: SGR 1806-20 - stars: individual: LBV 1806-20 - ISM: individual: W31 Galaxy: structure - Galaxy: kinematics and dynamics

\section{Introduction}

W31 is one of the largest $\mathrm{H}$ II complexes in the Galaxy, with intense star-forming regions that have been observed from radio to near-infrared wavelengths (e.g. Ghosh et al. 1989; Blum et al. 2001; Kim \& Koo 2002). At low spatial resolution, W31 appears as three main extended H II regions: G10.2-0.3, G10.3-0.1 and G10.6-0.4 (Shaver \& Goss 1970) (Fig. 1). The radio nebula G10.0-0.3 lies within W31 on the plane of the sky (Fig. 1), and has drawn considerable attention in recent years due to the intriguing objects nearby. At one time, G10.0-0.3 was suggested to be a plerionic supernova remnant powered by a rare soft gamma-ray repeater, SGR 1806-20 (Kulkarni \& Frail 1993; Kouveliotou et al. 1998). SGR 1806-20, in turn, was thought to be associated with an almost equally rare luminous blue variable (LBV) star (van Kerkwijk et al. 1995) which lies at the time-variable (in both flux and morphology) core of this nebula (Vasisht et al. 1995). However, the revised Inter-Planetary Network (IPN)

\section{Send offprint requests to: S. Corbel,}

e-mail: corbel@discovery.saclay.cea.fr

^ Visiting astronomer, Cerro Tololo Inter-American Observatory, National Optical Astronomy Observatories, which are operated by the Association of Universities for Research in Astronomy, under contract with the National Science Foundation. localization of SGR 1806-20 provides a position inconsistent (see Fig. 1) with that of the LBV star and radio core of G10.0-0.3 (Hurley et al. 1999), though the LBV position is consistent with the radio core within the uncertainties $\left(\sim 2^{\prime \prime}\right)$. Recent Chandra and infrared observations confirm that the SGR lies $\sim 12^{\prime \prime}$ away from the LBV and radio core (Eikenberry et al. 2001; Kaplan et al. 2002). Furthermore, Gaensler et al. (2001) argue that G10.0-0.3 is not a supernova remnant at all, but is rather powered by the tremendous wind of the LBV star at its core. Infrared observations of the field of SGR 1806-20 reveal that the LBV star is not alone, but appears to be part of a cluster of embedded, hot, luminous stars (Fuchs et al. 1999), and the IPN position for SGR 1806-20 is consistent with membership in the star cluster (Eikenberry et al. 2001). Given this somewhat confusing history, we take a moment to summarize our current understanding of G10.0-0.3: 1 . G10.0-0.3 is a radio nebula (NOT supernova remnant) with emission powered by the LBV star spatially coincident with its core. 2. The LBV star is part of a cluster of luminous stars embedded in a molecular cloud. 3 . SGR $1806-20$ is likely another member of this cluster of stars, and is spatially distinct from the LBV star. We plot in Fig. 1 the various objects (and their relative location on the plane of the sky) that we will discuss in this paper. 


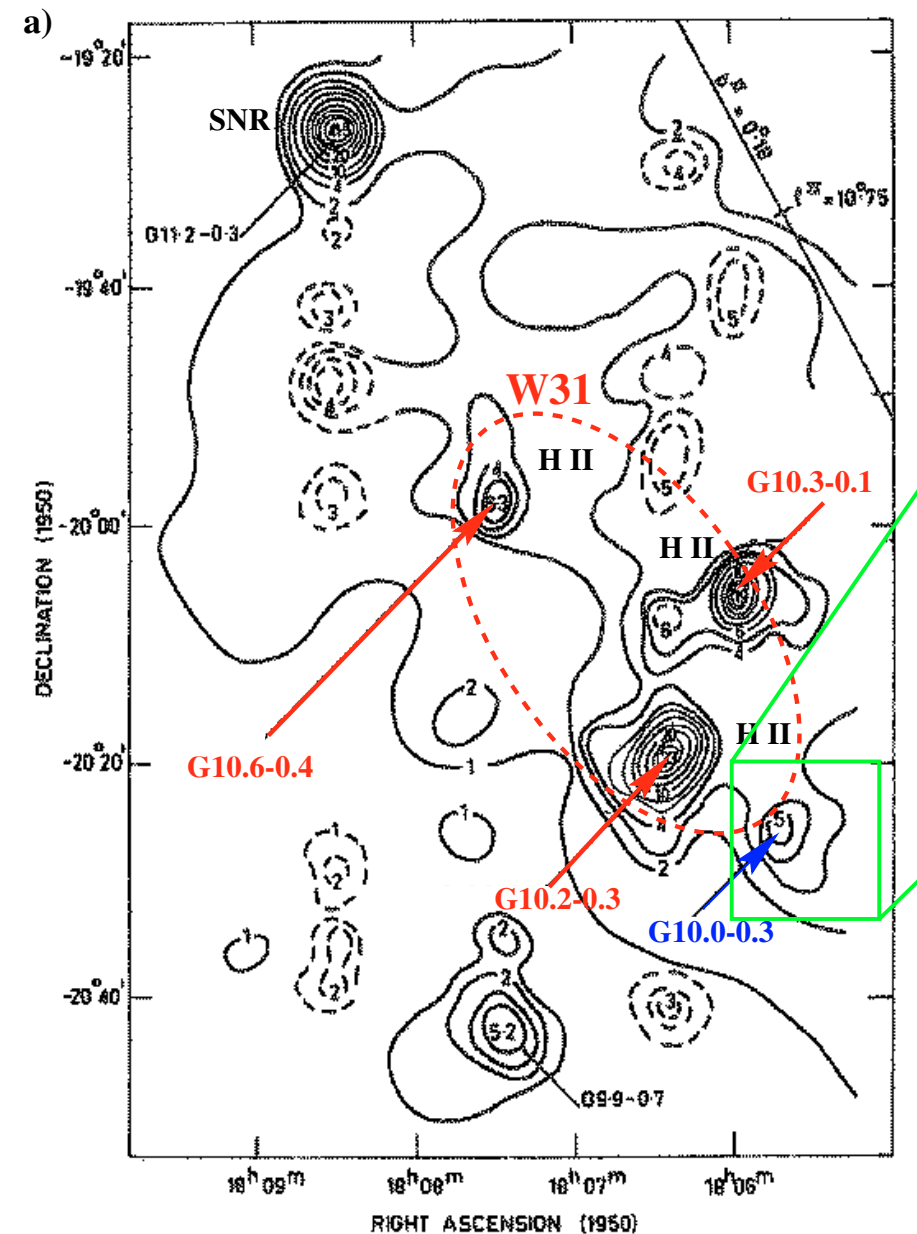

b)

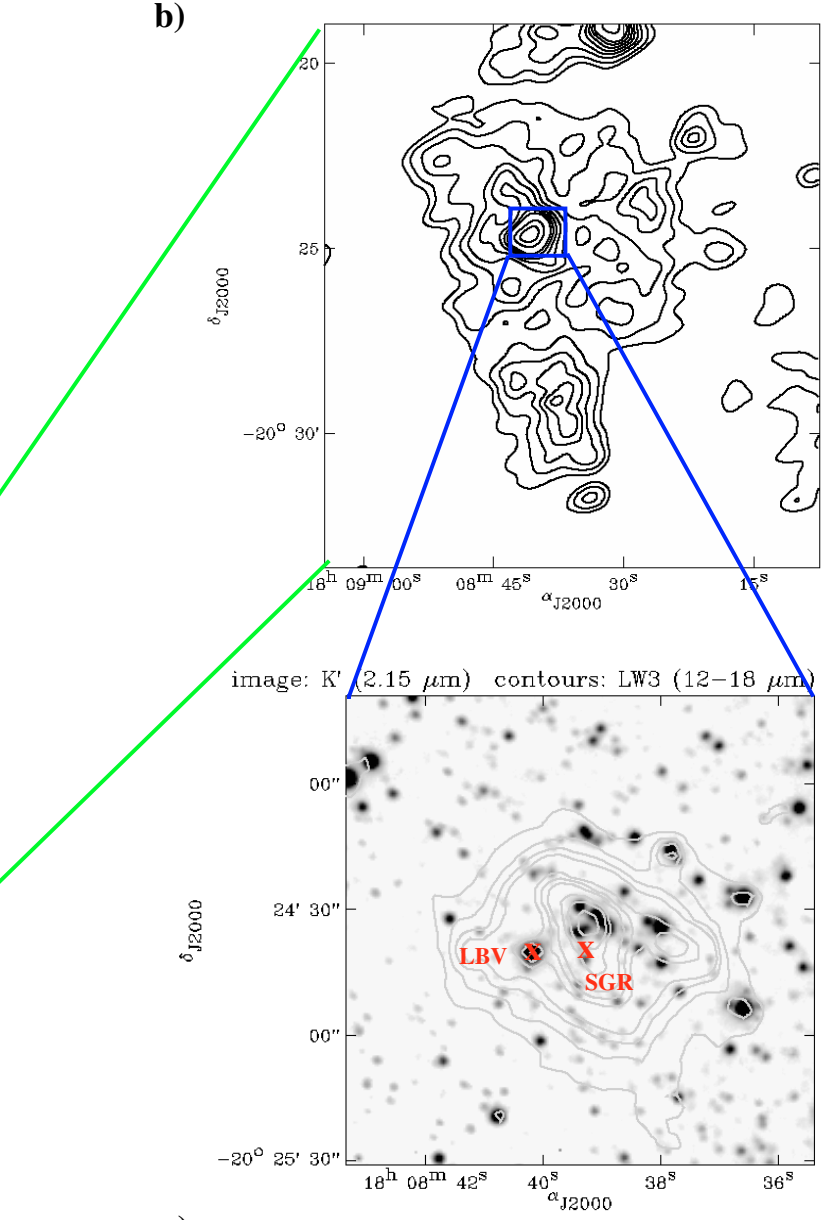

c)

Fig. 1. Representation of the location of each object introduced in this paper and their relative positions on the plane of the sky. a) Large radio map at $408 \mathrm{MHz}$ of the field of W31, adapted from Shaver \& Goss (1970). The location of the main components of W31 are labeled, as well as the radio nebula G10.0-0.3 (produced by the wind of LBV 1806-20). b) Radio image of the radio nebula G10.0-0.3 at 1.4 GHz from Kulkarni et al. (1994). c) $K^{\prime}(2.15 \mu \mathrm{m})$ near-infrared image $\left(\sim 2^{\prime} \times 2^{\prime}\right)$ of the core of G10.0-0.3. The location of LBV 1806-20 and SGR 1806-20 are indicated by a cross. The ISOCAM LW3 $(12-18 \mu \mathrm{m})$ contours are superposed on this image in order to highlight the spatial extend of the cluster of massive stars around SGR 1806-20 (adapted from Fuchs et al. 1999).

Because of the proximity of these unusual objects, the distance to them can provide significant insight into their physical properties, giving this measurement particular importance. Corbel et al. (1997) proposed a distance estimate based on observations of molecular clouds along the line of sight. They used CO spectroscopy to estimate the hydrogen column density towards G10.0-0.3, and from that an absorption column density as a function of distance. Taking the measured X-ray absorption towards SGR 1806-20 and an estimate of the optical extinction to the LBV star, they concluded that G10.0-0.3, SGR 1806-20 and W31 lie $14.5 \pm 1.4 \mathrm{kpc}$ from the Sun.

However, this situation seemed complicated by newer infrared stellar spectroscopy by Blum et al. (2001) and radio/millimeter observations by Kim \& Koo (2002). Blum et al. (2001) present infrared spectra of members of a star cluster in the H II region G10.2-0.3, also within W31 on the plane of the sky. Based on the spectra, they derive spectral/luminosity classes and extinctions for the stars, which, combined with infrared photometry, place them and G10.2-0.3 unambiguously at a distance of $d \simeq 3.4 \mathrm{kpc}$. However, the extinction towards G10.2-0.3 is much smaller $\left(\triangle A_{\mathrm{V}} \approx\right.$ $15 \mathrm{mag}$ ) than the extinction towards LBV 1806-20 and the star cluster close to SGR 1806-20 (Eikenberry et al. 2001) and the correlated X-ray absorption towards SGR 1806-20 (Mereghetti et al. 2000). Furthermore, 21-cm continuum and several molecular line maps of the region (Kim \& Koo 2002) show that G10.0-0.3 is rather separated from the primary components of W31 (G10.2-0.3 and G10.3-0.1). Thus, as of this writing, there is no definitive physical linkage between G10.0-0.3 and the major components of W31. Together, these imply that G10.0-0.3 may lie at a different distance along the line of sight than G10.2-0.3. It is this new, morecomplicated situation which motivates us to reconsider the distance to G10.0-0.3 (and by extension LBV 1806-20 and SGR 1806-20) in the light of previous work by Corbel et al. (1997) and Blum et al. (2001) as well as new observations.

In this paper, we present newer, higher velocity - resolution CO spectroscopy towards LBV 1806-20 and its associated radio nebula G10.0-0.3, and also towards 2 (out of 3 ) of 

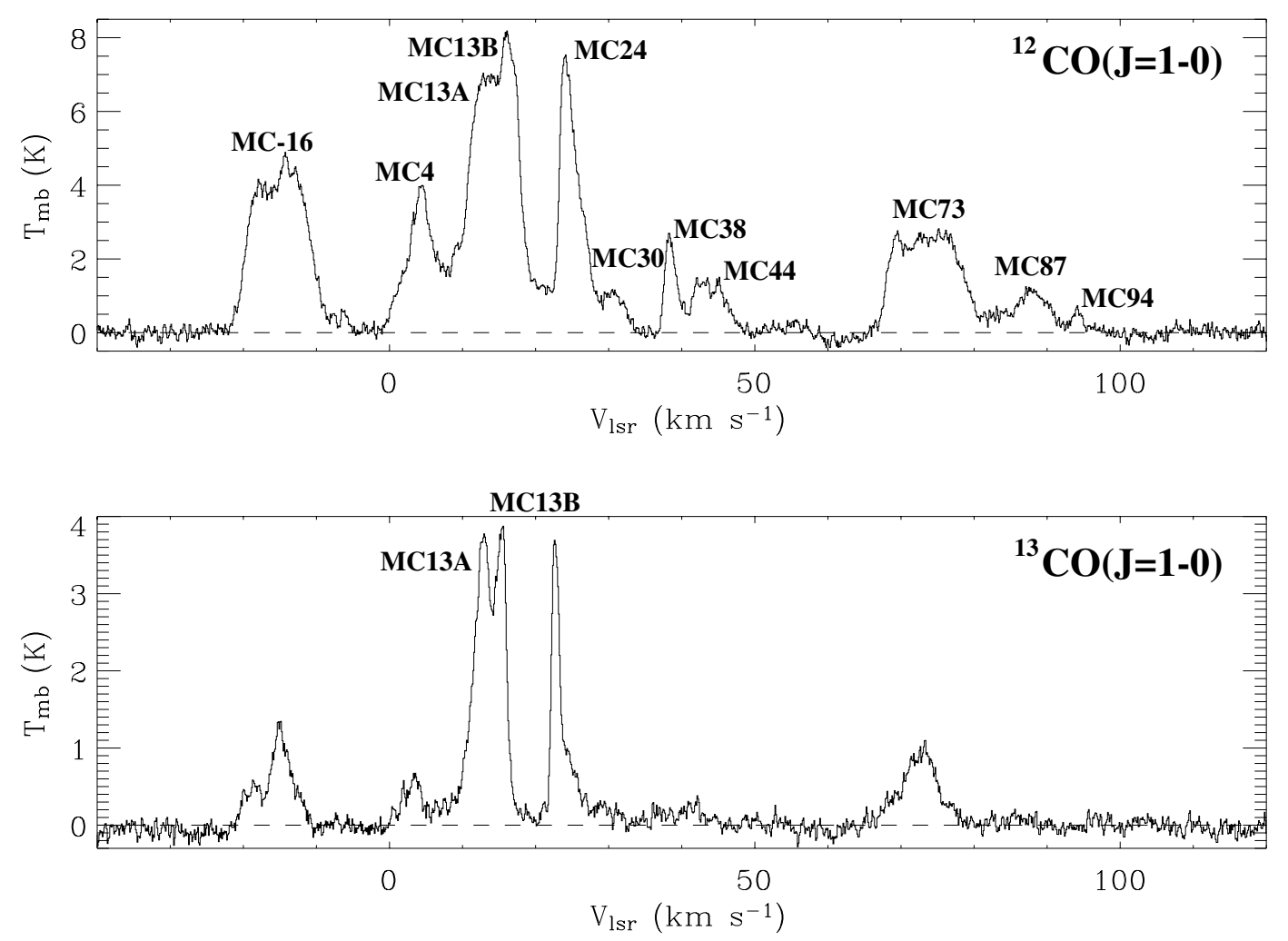

Fig. 2. ${ }^{12} \mathrm{CO}(J=1-0)($ top $)$ and ${ }^{13} \mathrm{CO}(J=1-0)$ (bottom) spectrum in the direction of LBV 1806-20 and SGR 1806-20. Antenna temperatures have been converted into main beam brightness temperatures. Each molecular cloud is labeled by its name.

the brightest $\mathrm{H}$ II regions of $\mathrm{W} 31$. We also present the $\mathrm{NH}_{3}$ absorption spectrum originally mentioned in Corbel et al. (1997). We then add higher-resolution infrared spectroscopic observations of the LBV star at the center of G10.0-0.3. In Sect. 2, we present the observations and data reduction as well as the main results from our observations. In Sect. 3, we use these data to derive a robust distance estimate for LBV 1806-20 and G10.0-0.3. We then discuss in some detail the implications of this distance for SGR 1806-20 and LBV 1806-20, as well as the structure of W31 as a whole. Finally, in Sect. 4 we present our conclusions.

\section{Observations}

\subsection{CO spectra}

Following the work by Corbel et al. (1997), we obtained new millimeter observations with the $15 \mathrm{~m}$ Swedish-ESO Submillimeter Telescope (SEST) at La Silla, Chile, on 1998 August 27 and 1999 March 2. We took spectra at the position of LBV 1806-20 (same as in Corbel et al. 1997) at the transitions ${ }^{12} \mathrm{CO}(J=1-0)$ and ${ }^{13} \mathrm{CO}(J=1-0)$ for a total integration time of $5 \mathrm{~min}$ each (Fig. 2). As the fullwidth at half-maximum $(F W H M)$ beamwidth of the SEST is $45^{\prime \prime}$ at $\sim 115 \mathrm{GHz}$, the spectra toward LBV 1806-20 also include the region of SGR $1806-20$. Additionally, at ${ }^{12} \mathrm{CO}(J=$ 1-0) we made spectral observations towards two $\mathrm{H}$ II regions of W31 (G10.2-0.3 and G10.3-0.1) with an integration time of 2 min each.
We acquired the spectra in position-switching mode which consists of switching (every one minute) between sources and an off-position free of emission. We then averaged the spectra after baseline removal. The back end was an acousto-optical spectrometer with 2000 channels and a frequency bandwidth of $86 \mathrm{MHz}$, giving a high velocity resolution of $0.11 \mathrm{~km} \mathrm{~s}^{-1}$ (to be compared with the velocity resolution of $2.3 \mathrm{~km} \mathrm{~s}^{-1}$ in Corbel et al. 1997). The receiver was calibrated with the standard chopper-wheel method. Systems temperature during the observations were typically in the range $200-350 \mathrm{~K}$. We converted all $\mathrm{CO}$ spectra into main-beam brightness temperature $\left(\eta_{\text {eff }}=0.7\right)$, which is expressed in term of radial velocity in the Local Standard of Rest (LSR).

We note that there is a distance ambiguity for any molecular cloud within the solar circle at a given radial velocity (in the LSR). Indeed, at each radial velocity it is possible to locate the cloud on the near side (near distance) or on the far side (far distance) of the Galaxy. We assume an error of $10 \mathrm{~km} \mathrm{~s}^{-1}$ for the velocity-distance conversion using the rotation curve of the Galaxy of Fich et al. (1989) with the standard rotation constants of $R_{0}=8.5 \mathrm{kpc}$ and $\Theta_{0}=220 \mathrm{~km} \mathrm{~s}^{-1}$. This is sufficiently large to cover any velocity deviation $\left(\sim 4 \mathrm{~km} \mathrm{~s}^{-1}\right)$ with respect to the LSR frame of Galactic rotation (Combes 1991). This implies that the error bars associated to the distances are probably consistent with $2 \sigma$ confidence levels.

The ${ }^{12} \mathrm{CO}$ spectra (Fig. 2) toward LBV 1806-20 reveal a complex line of sight, as already discussed in Corbel et al. (1997), with various molecular clouds detected (see Table 1 
Table 1. Derived parameters for each of the molecular clouds along the line of sight of LBV $1806-20$ and SGR $1806-20$, using the ${ }^{12} \mathrm{CO}(J=$ $1-0)$ spectra.

\begin{tabular}{|c|c|c|c|c|c|c|}
\hline Name & $\begin{array}{c}W\left(\mathrm{CO}^{*}\right)^{a} \\
\left(\mathrm{~K} \mathrm{~km} \mathrm{~s}^{-1}\right)\end{array}$ & $\begin{array}{c}V_{\mathrm{lsr}}^{b} \\
\left(\mathrm{~km} \mathrm{~s}^{-1}\right)\end{array}$ & $\begin{array}{c}A_{\mathrm{V}} \\
(\mathrm{mag})\end{array}$ & $\begin{array}{c}\text { Near distance }{ }^{b} \\
(\mathrm{kpc})\end{array}$ & $\begin{array}{c}\text { Far distance }{ }^{b} \\
(\mathrm{kpc})\end{array}$ & $\begin{array}{c}\text { Distance }^{b} \\
(\mathrm{kpc})\end{array}$ \\
\hline $\mathrm{MC}-16$ & 28.4 & -14.9 & $8.6 \pm 1.7$ & n.a. & 24.9 & 4.5 \\
\hline MC 4 & 12.7 & 4.3 & $3.9 \pm 0.8$ & 0.2 & 16.6 & 0.2 \\
\hline MC 13A & 32.8 & 12.7 & $10.0 \pm 2.0$ & 1.7 & 15.1 & 15.1 \\
\hline MC 13B & 9.8 & 16.7 & $3.0 \pm 0.6$ & 2.2 & 14.5 & 4.5 \\
\hline MC 24 & 18.3 & 23.6 & $5.6 \pm 1.1$ & 3.0 & 13.8 & 3.0 \\
\hline MC 30 & 2.6 & 30.0 & $0.8 \pm 0.2$ & 3.5 & 13.2 & 3.5 \\
\hline MC 38 & 3.6 & 38.4 & $1.1 \pm 0.2$ & 4.2 & 12.6 & 4.2 \\
\hline MC 44 & 5.0 & 43.5 & $1.6 \pm 0.3$ & 4.5 & 12.3 & 4.5 \\
\hline MC 73 & 19.8 & 73.4 & $6.0 \pm 1.2$ & 5.7 & 11.0 & $\ldots{ }^{c}$ \\
\hline MC 87 & 4.5 & 87.9 & $1.4 \pm 0.3$ & 6.1 & 10.6 & $\ldots^{c}$ \\
\hline MC 94 & 1.7 & 94.2 & $0.5 \pm 0.1$ & 6.2 & 10.5 & $\ldots{ }^{c}$ \\
\hline
\end{tabular}

${ }^{a} W(\mathrm{CO} *)$ is the integrated line area of the cloud. The error on $W\left(\mathrm{CO}^{*}\right)$ itself is negligeable compared to the $20 \%$ uncertainty we assume to take into account the uncertainties in the various conversion factors for $A_{\mathrm{V}}$.

${ }^{b}$ In the text, we use a systematic error of $\pm 10 \mathrm{~km} \mathrm{~s}^{-1}$ on the velocity of the cloud in order to derive a robust distance range (>2 $\sigma$ ) for each of the molecular clouds.

${ }^{c}$ We did not attempt to resolve the distance ambiguity for MC 73, MC 87 and MC 94, since they are between MC -16 and MC 13A in either case.

for detailed informations). Due to the high velocity resolution, some of the molecular clouds mentioned in Corbel et al. (1997) are now split into two components; for simplicity the same notation is kept as in Corbel et al. (1997). MC 38 is decomposed into the component at $\sim 38 \mathrm{~km} \mathrm{~s}^{-1}$ and a new cloud at $\sim 44 \mathrm{~km} \mathrm{~s}^{-1}$ (now noted as MC 44). The resolution of the edge of the ${ }^{12} \mathrm{CO}$ line of MC 24 also reveals a new cloud at $30 \mathrm{~km} \mathrm{~s}^{-1}$ (MC 30). MC 87 is now split into MC 87 and $\mathrm{MC}$ 94. However, the most interesting feature in the new ${ }^{12} \mathrm{CO}(J=1-0)$ spectrum toward LBV $1806-20$ is the splitting of molecular cloud MC 13 into two components. This is confirmed by the ${ }^{13} \mathrm{CO}(J=1-0)$ transition (an optically thin line, with narrower $F W H M$ ), which clearly reveals that MC 13 is resolved into two components (Fig. 2): one at $13.0 \pm 0.1 \mathrm{~km} \mathrm{~s}^{-1}$ (hereafter MC 13A) and one at $15.5 \pm 0.1 \mathrm{~km} \mathrm{~s}^{-1}$ (hereafter MC 13B). (These velocities are obtained if we naively fit the lines with Gaussian profiles.) This also implies that the shape of the ${ }^{12} \mathrm{CO}(J=1-0)$ spectrum around $13 \mathrm{~km} \mathrm{~s}^{-1}$ is not due to opacity effects. Fitting the ${ }^{12} \mathrm{CO}(J=1-0)$ spectra leads to velocities of $12.7 \pm 0.1 \mathrm{~km} \mathrm{~s}^{-1}$ and $16.7 \pm 0.1 \mathrm{~km} \mathrm{~s}^{-1}$ for MC $13 \mathrm{~A}$ and $\mathrm{MC}$ 13B respectively.

According to Kim \& Koo (2001), the velocity of the recombination line at the core of G10.2-0.3 is $16.4 \pm 0.2 \mathrm{~km} \mathrm{~s}^{-1}$, and it is therefore apparent that G10.2-0.3 is associated with the molecular cloud we labeled MC 13B. We remind the reader that in addition to the association in velocity, the map of the CO emission (Corbel et al. 1997) also points to an association of G10.2-0.3 with one of the clouds in this velocity range. We note that the velocity of the recombination line of the other major H II region studied by Kim \& Koo, G10.3-0.1, is $7.7 \pm 0.5 \mathrm{~km} \mathrm{~s}^{-1}$, with a range from 7.7 to $11.9 \mathrm{~km} \mathrm{~s}^{-1}$ (Kim \& Koo 2001), which is more consistent with the velocity of MC 13A.

\section{2. $\mathrm{NH}_{3}$ spectrum}

In addition to the above $\mathrm{CO}$ observations, we also include an $\mathrm{NH}_{3}$ absorption spectrum (Fig. 3) towards the radio nebula G10.0-0.3 (produced by the wind of LBV 1806-20) at the same position as the $\mathrm{CO}$ observations. This spectrum was originally mentioned, but not shown, in Corbel et al. (1997) as a "Note added in manuscript". As it is important for this work, we include it here. The $\mathrm{NH}_{3}$ observations (total integration time of $30 \mathrm{~min}$ ) were performed at the frequency of $23694.49 \mathrm{MHz}$ (velocity resolution of $0.5 \mathrm{~km} \mathrm{~s}^{-1}$ ) with the NASA Deep Space Network Goldstone 34-m antenna located in California, USA, and giving a beamwidth of $1.6^{\prime}$. As the radio continuum against which the $\mathrm{NH}_{3}$ absorption spectrum is measured is produced by the radio nebula G10.0-0.3, any absorption feature results from gas located in front of LBV 1806-20.

The most striking feature from this spectrum is the absorption line (detected at a significance level of $8.6 \sigma$ ) at a velocity of $70.9 \pm 0.5 \mathrm{~km} \mathrm{~s}^{-1}$, in full agreement with the $\mathrm{CO}$ velocity of the molecular cloud MC 73 that is detected along the line of sight to LBV 1806-20 (see Table 1). This detection thus demonstrates that the molecular cloud MC 73 is located in front of LBV 1806-20. The absorption line $(4.2 \sigma)$ at $\sim 62 \mathrm{~km} \mathrm{~s}^{-1}$ may be related to an $\mathrm{H}$ I shell around MC 73 or to a different H I cloud (cf. Fig. 1 in Corbel et al. 1997) in front of G10.0-0.3. A possible weaker absorption feature $(2.2 \sigma)$ 


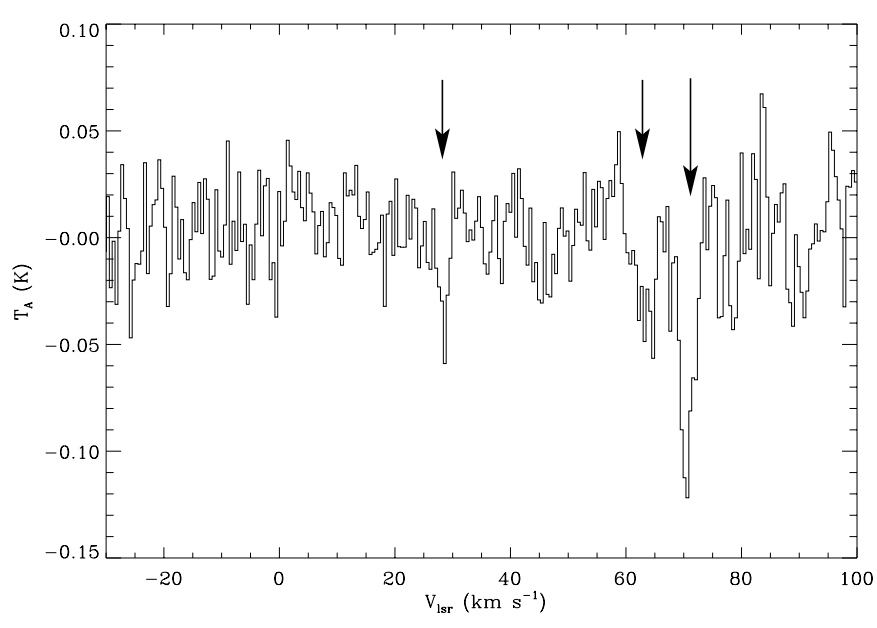

Fig. 3. $\mathrm{NH}_{3}$ absorption spectrum measured against the radio nebula G10.0-0.3 produced by the wind of LBV 1806-20. Arrows indicate the main $(\sim 9 \sigma)$ absorption feature at $71 \mathrm{~km} \mathrm{~s}^{-1}$ as well as the possible features at $62 \mathrm{~km} \mathrm{~s}^{-1}(\sim 4 \sigma)$ and $29 \mathrm{~km} \mathrm{~s}^{-1}(\sim 2 \sigma)$.

might also be present at $28.6 \mathrm{~km} \mathrm{~s}^{-1}$, which would be in agreement with the velocity of the MC 30 cloud and would indicate that MC 30 may also lie front of LBV 1806-20.

\subsection{IR spectra}

We used the Ohio State InfraRed Imaging Spectrograph (OSIRIS) instrument (Depoy et al. 1993) and f/14 tip-tilt secondary on the Cerro-Tololo Inter-American Observatory (CTIO) 4-meter telescope on July 5, 2001 to obtain moderate resolution ( $R=3000$ for 2 pixels) spectra of LBV 1806-20 in the $J, H$, and $K$ bands $(1-2.4 \mu \mathrm{m})$. We present details of these observations and their reduction elsewhere (Eikenberry et al. 2003), and present a reduced $K$-band spectrum in Fig. 4.

The colors of LBV 1806-20 (van Kerkwijk et al. 1995) and its spectral continuum shape allow us to estimate the extinction towards LBV 1806-20. For such a hot star (as indicated by the He I $2.112 \mu \mathrm{m}$ absorption feature - van Kerkwijk et al. 1995; Eikenberry et al. 2003), the intrinsic $J-K$ color is nearly neutral, and the observed red color of $J-K=5.0 \pm 0.15 \mathrm{mag}$ corresponds to an extinction of $A_{\mathrm{V}}=28 \pm 2$ mag (assuming the Rieke-Lebofsky reddening law, Rieke \& Lebofsky 1985), matching the estimates based on $\mathrm{CO}$ observations (Corbel et al. 1997). (While a hypothetical near-infrared excess from LBV 1806-20 would alter these conclusions, Eikenberry et al. (2003) show that this is not present, based on the spectral continuum shape across $J H K$ bands, and the close match in $J-K$ color between LBV 1806-20 and other cluster stars.) In addition, the $H$ and $K$ bands are in the Rayleigh-Jeans portion of the blackbody emission curve (the reason for the neutral colors noted above). Thus, we can estimate the extinction towards LBV 1806-20 by de-reddening the spectra until the continuum shape matches a Rayleigh-Jeans distribution. In this way, we obtain estimates of $A_{\mathrm{V}}=31 \pm 3$ mag from the $H$-band continuum and $A_{\mathrm{V}}=28 \pm 3 \mathrm{mag}$ from the $K$-band continuum, with uncertainties dominated by $\sim 10 \%$ uncertainty in the spectrograph response shape over a given waveband. Combining

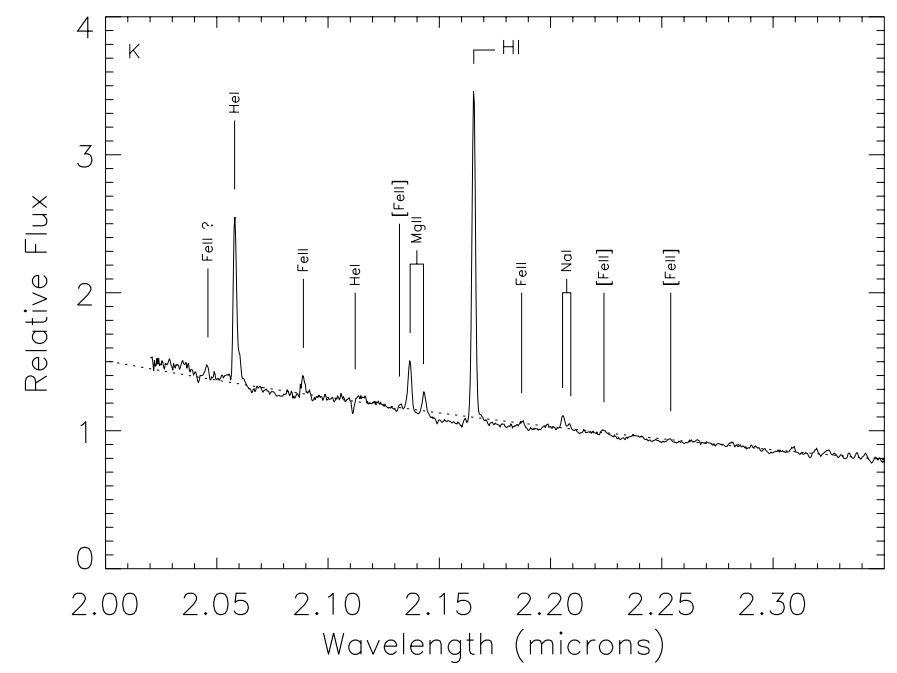

Fig. 4. Near-infrared spectrum of LBV 1806-20 in the $K$ band, dereddened with $A_{\mathrm{V}}=29 \mathrm{mag}$, following the reddening law of Rieke \& Lebofsky (1985). The dotted line indicates the spectral shape of a blackbody with $T>12000 \mathrm{~K}$.

these with the extinction estimate from the $J-K$ color above, we adopt a final estimate for the extinction of $A_{\mathrm{V}}=29 \pm 2 \mathrm{mag}$ towards LBV 1806-20.

From the emission lines, we can also measure a radial velocity for LBV 1806-20. We selected the Br $\gamma$ line as a velocity fiducial, as it is the strongest line detection in the spectrum, and appears to be relatively free from contamination due to blending with other strong lines. We fit a Gaussian profile to this line, finding no significant residuals, and a centroid shifted from the atmospheric rest frame by $-3 \pm 20 \mathrm{~km} \mathrm{~s}^{-1}$, where residuals in the spectral wavelength solution from atmospheric $\mathrm{OH}$ emission lines dominate the largely systematic uncertainty. After correcting for the Earth's barycentric motion and the Solar System barycenter motion relative to the local standard of rest, we determine a radial velocity for LBV 1806-20 of $v_{\mathrm{lsr}}=$ $10 \pm 20 \mathrm{~km} \mathrm{~s}^{-1}$. Cross-checks of this velocity determination with several other strong unblended lines in the spectra give consistent results for the velocity of LBV 1806-20. This velocity is important, as massive stars such as LBVs are a kinematically "cold" population, which do not generally deviate significantly in their velocities from their parent molecular clouds.

\section{Results and discussion}

\subsection{The distance to LBV 1806-20 and its associated radio nebula G10.0-0.3}

\subsection{1. $\mathrm{NH}_{3}$ absorption}

A critical point for measuring the distance to LBV 1806-20 is the detection of the $\mathrm{NH}_{3}$ absorption features (Fig. 3) against the radio continuum (G10.0-0.3) produced by the LBV star. A firm lower limit on the distance to G10.0-0.3 and LBV 1806-20 is the near distance associated with the absorption feature at $70.9 \pm 0.5 \mathrm{~km} \mathrm{~s}^{-1}$, i.e. $5.7 \pm 0.4 \mathrm{kpc}$. We can then combine the full velocity range $\left(-10\right.$ to $\left.30 \mathrm{~km} \mathrm{~s}^{-1}\right)$ for LBV 1806-20 
(Sect. 2.3 above) and the fact that LBV 1806-20 has to be located behind MC 73, together with the rotation curve of the Galaxy (Fich et al. 1989) to constrain the distance range for LBV 1806-20 without making any further assumptions regarding other molecular clouds along the line of sight. This straightforward calculation unambiguously places LBV 1806-20 in the distance range 13.2-21.5 kpc.

\subsection{2. $M C$ 13A, $M C 13 B$, and $G 10.2-0.3$}

This determination of the distance to G10.0-0.3, while unambiguous, differs significantly from the distance determination for the nearby (on the sky) stellar cluster G10.2-0.3, which Blum et al. (2001) place at $3.4 \pm 0.3 \mathrm{kpc}$. Given the similar radial velocities of LBV 1806-20 and G10.2-0.3 $\left(\sim 10-16 \mathrm{~km} \mathrm{~s}^{-1}\right)$, this seems somewhat surprising. However, on the plane of the sky, they are separated by $10.7^{\prime}$. Furthermore, the measured optical extinction of LBV 1806-20 $(29 \pm 2$ mag, Sect. 2.3) and G10.2-0.3 (15.5 $\pm 1.7 \mathrm{mag}$, Blum et al. 2001) are clearly not in agreement. This indicates that these two objects cannot be located at the same distance. G10.2-0.3, with a lower optical extinction, has to be located in the foreground relative to LBV 1806-20. Furthermore, Lavine et al. (2003) find that the stellar field surrounding LBV 1806-20 includes two distinct populations of stars - one with $A_{\mathrm{V}} \sim 30$ mag (consistent with LBV 1806-20) and another with $A_{\mathrm{V}} \sim 15 \mathrm{mag}$ (consistent with G10.2-0.3). Fuchs et al. (1999) also find the same bimodality in extinction, albeit with a much smaller sample. The fact that no stars are found with intermediate extinctions demonstrates that these are distinct populations, rather than a single population suffering from differential extinction across the field. Thus, we are forced to conclude that the stellar cluster of Blum et al. (2001) is a distinct cluster in the foreground to the cluster containing LBV 1806-20. Thus, discrepant distances for these two distinct clusters are not surprising, and in fact should be expected.

In order to understand the fact that these distinct populations have similar radial velocities, we need to take into account (as outlined in Sect. 2.1) that the molecular cloud labelled MC 13 in Corbel et al. (1997) is now known to consist of two distinct components that we have called MC 13A and MC 13B. Thus, the line of sight towards these objects is much more complex than previously thought, with two molecular clouds with similar velocities, one containing each stellar population. Based on the fact that MC 13B is associated with G10.2-0.3 (Sect. 2.1; Kim \& Koo 2002), and that the upper limit for the distance to G10.2-0.3 (Blum et al. 2001) is much less than the $13.2 \mathrm{kpc}$ lower limit for LBV 1806-20, we conclude that the molecular cloud MC $13 \mathrm{~B}$ is located in the foreground relative to LBV 1806-20.

We can further constrain the parent molecular cloud of LBV 1806-20 by investigating the complex of molecular clouds along the line of sight. Absorption lines against the radio continuum of G10.2-0.3 (see Corbel et al. 1997, and references therein) showed that MC 4, MC 24, MC 30, MC 38, MC 44 are located at their near distances. Given the ammonia absorption towards LBV 1806-20 and its accompanying distance range of 13-21 kpc, the only remaining molecular clouds along the line of sight which could potentially be the site of LBV 1806-20 are MC 87, MC 94, and MC 13A. Of these, only MC 13A has a velocity consistent with LBV 1806-20 (Sect. 2.3), and we conclude that it is the parent molecular cloud for LBV 1806-20, SGR 1806-20, and G10.0-0.3. This association and the distance range for LBV 1806-20 now lift the near/far distance ambiguity for MC 13A, and we can use the velocity of MC 13A and the Galactic rotation curve to constrain the distance for both MC 13A and LBV 1806-20 to be $15.1_{-1.3}^{+1.8} \mathrm{kpc}$.

\subsubsection{Extinction and distance}

We can cross-check the above distance estimation, using the measured extinction towards LBV 1806-20. This extinction arises primarily within the molecular clouds along the line of sight, and we can determine its value for each cloud using the CO line area. In order to convert the molecular emission spectra into equivalent optical extinction along the line of sight, we follow the method outlined in Corbel et al. (1999) or Chapuis \& Corbel (2003). We assume conservative errors of $20 \%$ (to take into account the uncertainties in the various conversion factors) for the contribution in terms of optical extinction of each molecular cloud. The discussion of Corbel et al. (1997) for the location of the various molecular clouds still holds with these new observations - we simply need to consider the revised distance of G10.2-0.3 and the splitting of MC 13. Separating the contribution of each of the two MC 13 clouds in terms of line area is not a simple task. But if we fit both ${ }^{12} \mathrm{CO}(J=1-0)$ and ${ }^{13} \mathrm{CO}(J=1-0)$ spectra with two Gaussian lines in the velocity range $8-20 \mathrm{~km} \mathrm{~s}^{-1}$, we find that MC 13B and MC 13A contribute roughly $23 \%$ and $77 \%$ respectively in terms of line area. These should not be taken as firm numbers, but rather as indicative of the approximate relative contribution of each clouds. The resulting parameters (velocity, integrated area, optical extinction, distances) of the molecular clouds are presented in Table 1.

If we take the contribution from molecular hydrogen in all of the clouds up to $\mathrm{MC} 13 \mathrm{~A}$, we reach a total extinction of $32.5 \pm 2.6 \mathrm{mag}$. Taking the contribution from atomic hydrogen (Corbel et al. 1997) implies that the total optical extinction up to MC $13 \mathrm{~A}$ is $37.5 \pm 3.0 \mathrm{mag}$. While the uncertainties are not trivially small, this is at least consistent (at the $\sim 2 \sigma$ level) with the extinction of $29 \pm 2 \mathrm{mag}$ for LBV 1806-20. Adding the contribution of MC 13A significantly increases this extinction to $47.5 \pm 3.6 \mathrm{mag}$, implying that LBV $1806-20$ is at or close to the near side of MC 13A. In Fig. 5a, we present a map of the integrated ${ }^{13} \mathrm{CO}(J=1-0)$ emission (based on the data of Kim \& Koo 2002). It shows that the position of LBV 1806-20, as well as SGR 1806-20 and the star cluster, is consistent with a location on the edge of the cloud MC 13A, as favoured by the measurement of the optical extinction. Thus, we find a straightforward explanation for the distance of G10.0-0.3 and LBV 1806-20, consistent with all reported data, is that the star is located in MC $13 \mathrm{~A}$ at a distance of $15.1_{-1.3}^{+1.8} \mathrm{kpc}$. 


\subsection{The distance to G10.2-0.3 and the complex structure of W31}

\subsubsection{Distance and optical extinction of G10.2-0.3}

With the distance of G10.0-0.3 and LBV 1806-20 clarified, we now turn to the more general issues of the structure of W31, which we now understand to have several discrete components at distinct distances. In their study, Blum et al. (2001) performed near-infrared spectroscopy and photometry of an embedded stellar cluster in G10.2-0.3 (the other major $\mathrm{H}$ II regions of W31 being G10.3-0.1 and G10.6-0.4). They derived a spectrophotometric distance for G10.2-0.3 (and by extension for W31) by assuming either zero-age main-sequence or dwarf luminosity class for the stars of the cluster, obtaining distances of $3.1 \pm 0.3 \mathrm{kpc}$ and $3.7 \pm 0.3 \mathrm{kpc}$ respectively. They derived an average optical extinction to G10.2-0.3 of $A_{\mathrm{V}}=15.5 \pm 1.7 \mathrm{mag}$ (Blum et al. 2001). Clearly, this distance range, as well as the optical extinction, are not in agreement with our previous estimate for W31 (Corbel et al. 1997), in which we associate W31 with the far kinematic distance of MC 13. However, as shown above, we now understand that MC 13 and W31 itself are composed of multiple discrete components distributed along the line of sight. Thus, as noted above, the distance to $\mathrm{G} 10.2-0.3$, MC $13 \mathrm{~B}$, and their portion of $\mathrm{W} 31$ is a separate issue from G10.0-0.3, LBV 1806-20, SGR 1806-20, MC 13A, and their portion of W31. Nevertheless, our investigation of the latter can provide important insights into the distance to the former.

First, as a cross-check on the relative placement of molecular clouds along the line of sight in Table 1, we can compare the extinction of the stars in G10.2-0.3 to that expected from the clouds along the line of sight (as done above for LBV 1806-20). If we use the ${ }^{12} \mathrm{CO}(J=1-0)$ spectrum towards LBV 1806-20, the total visual extinction due to the molecular material located in front of MC 13B (MC 4, MC 24, MC 30, MC 38, MC 44, cf. Corbel et al. 1997) is $13.0 \pm 1.5 \mathrm{mag}$. Adding the contribution from atomic hydrogen (as in Corbel et al. 1997) raises this number to $14.5 \pm 1.8 \mathrm{mag}$, consistent with the above estimate of the optical extinction to G10.2-0.3 at the $\sim 1 \sigma$ level. This re-confirms the relative placement of the clouds in Table 1.

\subsubsection{G10.2-0.3: A location on the $-30 \mathrm{~km} \mathrm{~s}^{-1}$ spiral arm}

Given this, an important question remains if we want to fully understand the velocity field along this line of sight: what is the distance to the parental molecular cloud (MC 13B) of G10.2-0.3? A distance range of 2.8-4.0 kpc (Blum et al. 2001) would imply a velocity range of $22.0-38.0 \mathrm{~km} \mathrm{~s}^{-1}$, which is at best marginally consistent with the observed velocity of G10.2-0.3 $\left(16.4 \pm 0.2 \mathrm{~km} \mathrm{~s}^{-1}\right.$, Kim \& Koo 2001). Also, more importantly, absorption lines against the radio continuum of G10.2-0.3 are observed up to $43 \mathrm{~km} \mathrm{~s}^{-1}$ (e.g. Wilson 1974; Greisen \& Lockman 1979; Kalberla et al. 1982), which is very difficult to reconcile with G10.2-0.3 at the near distance of $2.2_{-1.6}^{+1.0} \mathrm{kpc}$ associated with the velocity of $16.4 \mathrm{~km} \mathrm{~s}^{-1}$.
Indeed, the cloud at the origin of the absorption at the velocity of $43 \mathrm{~km} \mathrm{~s}^{-1}$ is located at a distance greater than $4.5 \mathrm{kpc}$ (see Table 1) if we assume circular motion. But we would like to note that absorption at velocities greater than $\sim 50 \mathrm{~km} \mathrm{~s}^{-1}$ is not expected at these Galactic longitudes, because of a hole (Fig. 6) in the gas distribution (Dame et al. 1987; Corbel et al. 1997). The drop-off of absorption at $\sim 43 \mathrm{~km} \mathrm{~s}^{-1}$ is just the sharp inner edge of the molecular ring (Dame et al. 2001). Thus, the lack of absorption should not be an argument for ruling out a possible location at a far distance (e.g. Fish et al. 2003 for G10.6-0.4).

To overcome these difficulties, Blum et al. (2001), as well as other authors (e.g. Wilson 1974; Kalberla et al. 1982), invoked the presence of non-circular motion that could affect the velocity of the cloud (in addition to the contribution of rotation around the Galactic Center, hereafter GC). Indeed, along the line of sight of W31 (and LBV 1806-20) is found the 3-kpc expanding arm. This arm can be described as a simple rotating ring at a galactocentric radius of $3.4 \mathrm{kpc}$ (assuming a distance between the Sun and the GC of $8.5 \mathrm{kpc}^{1}$ ) expanding out from the GC with a velocity of $53 \mathrm{~km} \mathrm{~s}^{-1}$ (e.g. Bania 1980).

As discussed in detailed in Corbel et al. (1997), the 3-kpc expanding arm is likely associated with the molecular cloud MC -16 and is very likely not associated with MC 13B and G10.2-0.3. This is confirmed if we look at the radial velocity profile of the 3-kpc expanding arm as a function of the Galactic longitude based on H I observations (see Fig. 4 of Menon \& Ciotti 1970). This clearly show that a velocity of $\sim-15 \mathrm{~km} \mathrm{~s}^{-1}$ (as for MC -16) should be expected for this feature.

So, in order reconcile the fact that G10.2-0.3 is located on this side of the Galaxy (Blum et al. 2001) and the fact that absorption lines are observed up to a velocity of $43 \mathrm{~km} \mathrm{~s}^{-1}$, we surmise that there might be another feature with non-circular motion on this side of the Galaxy. In fact, there is another arm, the $-30 \mathrm{~km} \mathrm{~s}^{-1}$ spiral arm (unfortunately sometimes called the $4 \mathrm{kpc}$ arm), that is also expanding from the GC at a galactocentric radius of $4 \mathrm{kpc}$ (Menon \& Ciotti 1970; Greaves \& Williams 1994). Its expansion velocity measured at a Galactic longitude of $0^{\circ}$ is $-30 \mathrm{~km} \mathrm{~s}^{-1}$ (Menon \& Ciotti 1970; Liszt et al. 1977; Linke et al. 1981; Greaves \& Williams 1994; Sandqvist et al. 2003). This feature was originally detected in 1967 (Kerr \& Vallak 1967), but we would like to point that no molecular counterpart has been associated with it. Indeed, unlike the $3 \mathrm{kpc}$ expanding arm, the $-30 \mathrm{~km} \mathrm{~s}^{-1}$ spiral arm can not be traced on the longitude-velocity CO map (Fig. 6), possibly due to its proximity with the molecular ring (Dame et al. 2001). The extrapolation of its radial velocity profile in Fig. 1 of Menon \& Ciotti (1970) to a longitude of $10^{\circ}$ is consistent with the velocity of MC 13B. We therefore conclude that G10.2-0.3 and MC 13B are located on the $-30 \mathrm{~km} \mathrm{~s}^{-1}$ spiral arm. In that case, there is no problem with the fact that absorption lines against the radio continuum of G10.2-0.3 are detected up to $43 \mathrm{~km} \mathrm{~s}^{-1}$, as they would originate in MC 44 . We note that the $-30 \mathrm{~km} \mathrm{~s}^{-1}$ spiral arm has to be closer to the Sun than the 3-kpc expanding arm with a separation of $\sim 0.5 \mathrm{kpc}$

\footnotetext{
${ }^{1}$ Note that in old literature, this arm was sometimes called the "4-kpc expanding arm" due to a distance to the GC of $10.0 \mathrm{kpc}$.
} 

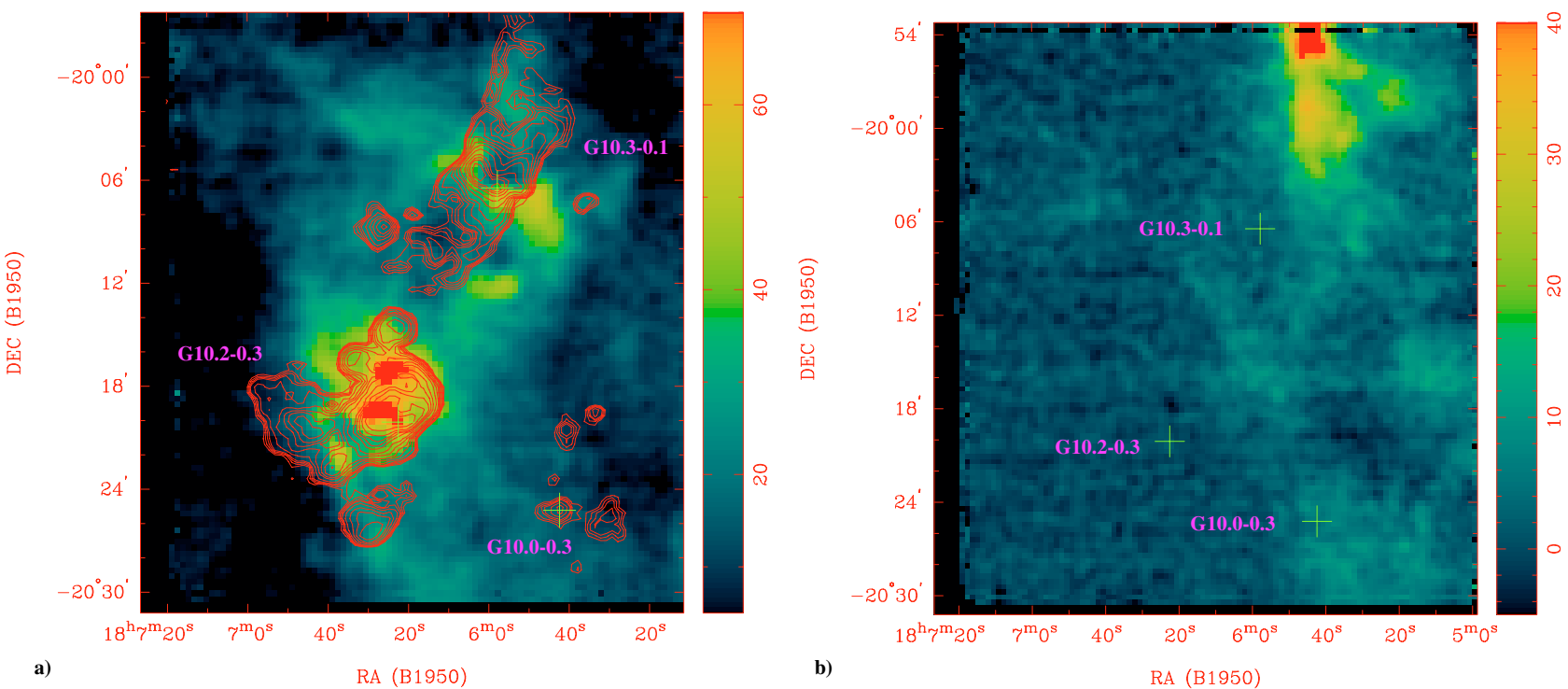

Fig. 5. a) Map of the ${ }^{13} \mathrm{CO}(J=1-0)$ emission integrated between the velocities 8 to $20 \mathrm{~km} \mathrm{~s}^{-1}$, using the data of Kim \& Koo (2002). b) Map of the ${ }^{13} \mathrm{CO}(J=1-0)$ emission integrated between the velocities 61 to $80 \mathrm{~km} \mathrm{~s}^{-1}$. The location of G10.2-0.3, G10.3-0.1 and G10.0-0.3 are marked. The unit of the scale of the map is in $\mathrm{K} \mathrm{km} \mathrm{s}^{-1}$. The contours represent the $21 \mathrm{~cm}$ radio continuum emission from this field of view (from Kim \& Koo 2002).

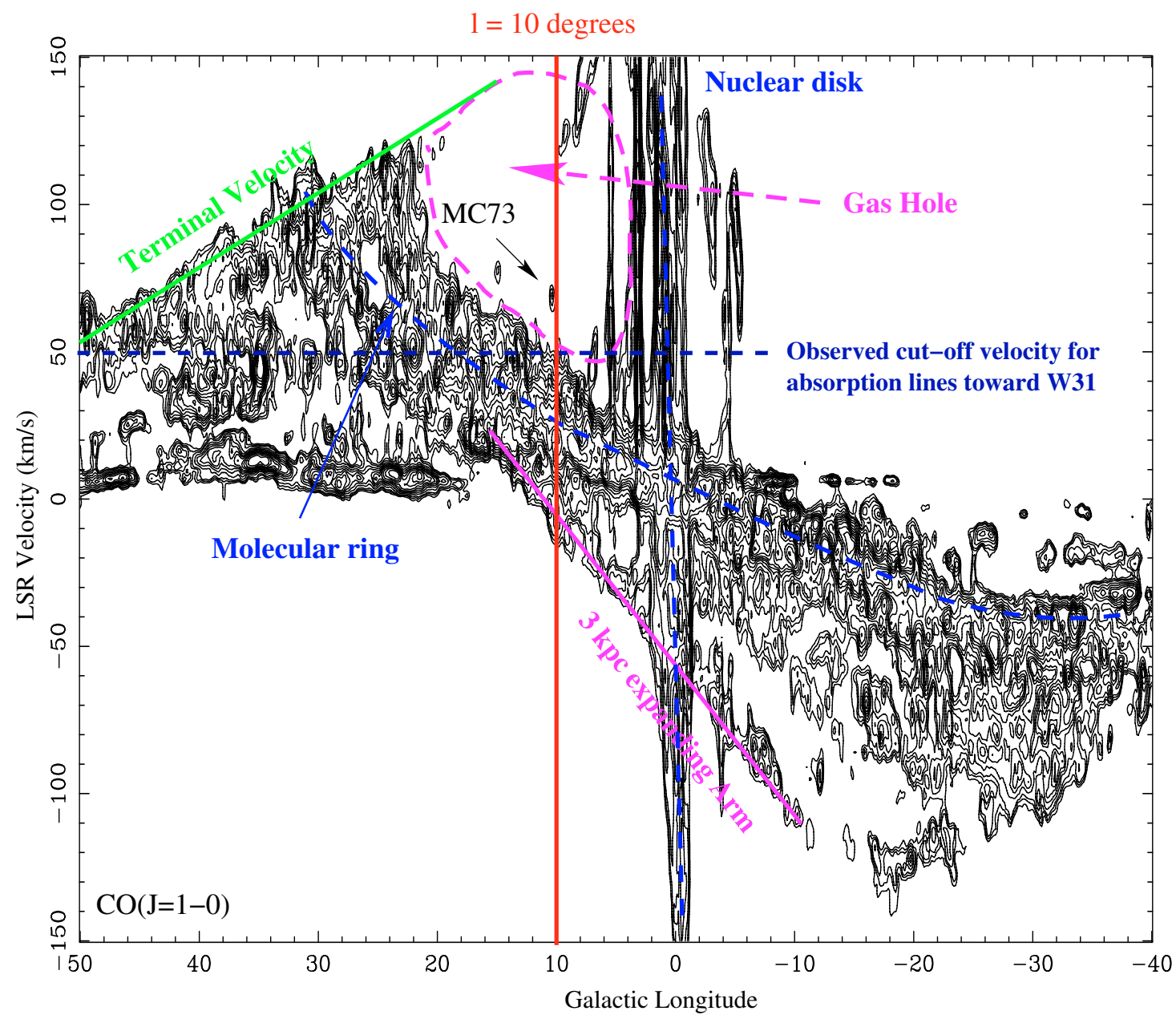

Fig. 6. Longitude - velocity map of Galactic CO emission, figure adapted from Dame et al. (2001). Between Galactic longitude of $\sim 5^{\circ}$ to $25^{\circ}$, a hole is apparent in the gas distribution at velocities greater than $\sim 50 \mathrm{~km} \mathrm{~s}^{-1}$. We note the location of MC 73 on the line of sight to G10.0-0.3. The locus point of the $3 \mathrm{kpc}$ expanding arm can also be well traced. 

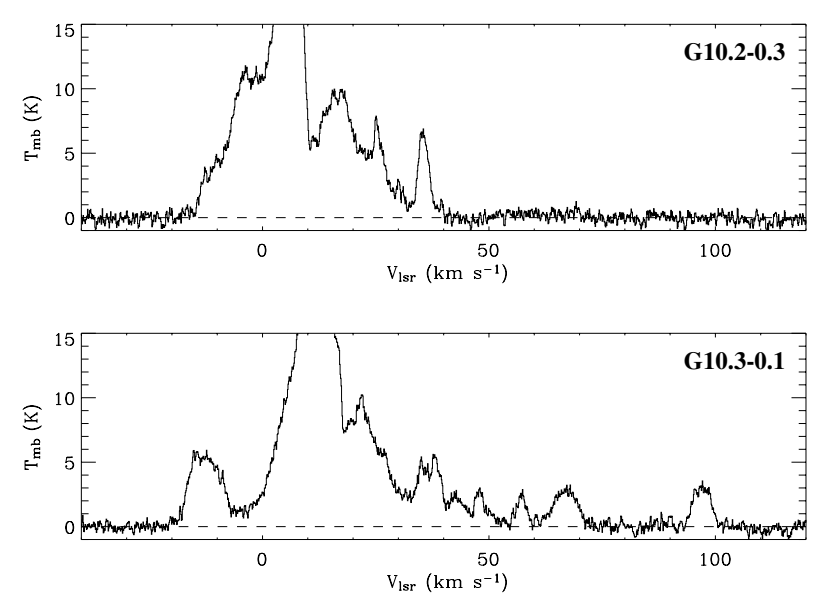

Fig. 7. ${ }^{12} \mathrm{CO}(J=1-0)$ spectrum in the direction of the $\mathrm{H}$ II regions G10.2-0.3 (top) and G10.3-0.1 (bottom). Antenna temperature have been converted into main beam brightness temperature. The scale has been adjusted in order to highlight the emission above $40 \mathrm{~km} \mathrm{~s}^{-1}$.

(Menon \& Ciotti 1970). In any case, the distance to the $-30 \mathrm{~km} \mathrm{~s}^{-1}$ spiral arm is set by the maximum velocity of the absorption lines, i.e. $4.5 \pm 0.6 \mathrm{kpc}$, which is completely consistent with the spectrophotometric distance of $3.4 \pm$ $0.6 \mathrm{kpc}$ for G10.2-0.3 (Blum et al. 2001). We note that at a Galactic longitude of $0^{\circ}$, the $3 \mathrm{kpc}$ expanding arm and the $-30 \mathrm{~km} \mathrm{~s}^{-1}$ spiral arm have a velocity separation of $\sim 23 \mathrm{~km} \mathrm{~s}^{-1}$, which is almost the difference in velocity between $\mathrm{MC}-16$ and MC 13B. This therefore strengthens our association of the $3-\mathrm{kpc}$ expanding arm with $\mathrm{MC}-16$ and the $-30 \mathrm{~km} \mathrm{~s}^{-1}$ spiral arm with MC 13B. We also note that these two arms could be related to the presence of a bar at the GC (e.g. Blitz \& Spergel 1991).

\subsubsection{The distance to $\mathrm{G} 10.3-0.1$ and $\mathrm{G} 10.6-0.4$}

So if G10.2-0.3 is at a closer distance, are the other major components of $\mathrm{W} 31$ at the same distance? In their detailed study of W31, Kim \& Koo (2002) performed a ${ }^{13} \mathrm{CO}(J=1-0)$ map of G10.2-0.3 and G10.3-0.1 (see their Figs. 4, 5 and 9). Their peak CO maps over the velocity range $0-22 \mathrm{~km} \mathrm{~s}^{-1}$ shows two main components (one centered on each $\mathrm{H}$ II region) that could be interpreted as two separate molecular clouds (see also Fig. 5a with the integrated ${ }^{13} \mathrm{CO}(J=1-0)$ map). But based on our new $\mathrm{CO}$ results, it might be possible that the southern part is associated with MC 13B as G10.2-0.3, and the northern part with MC 13A and G10.3-0.1 (Fig. 5a). If this is the case, it would imply that W31 could be decomposed into several components, with G10.3-0.1 located at the kinematic distance associated with MC $13 \mathrm{~A}$, i.e. $15.1_{-1.3}^{+1.8} \mathrm{kpc}$.

Figure 7 shows the ${ }^{12} \mathrm{CO}$ spectrum along the line of sight to these two $\mathrm{H}$ II regions. These profiles are very different, especially at velocities above $\sim 40 \mathrm{~km} \mathrm{~s}^{-1}$, and the profile of G10.3-0.1 is very similar to the one of LBV 1806-20 (the additional $\mathrm{CO}$ emission above $40 \mathrm{~km} \mathrm{~s}^{-1}$ may also be related to the variation in galactic latitude of these sources). So it is not unlikely that this $\mathrm{H}$ II region, which has a recombination line at $7.7 \pm 0.5 \mathrm{~km} \mathrm{~s}^{-1}$, could be associated with MC 13A. As noted above (Sect. 2.1), the ${ }^{13} \mathrm{CO}(J=1-0)$ map of this part of W31 by Kim \& Koo (2002) could be interpreted as being due to the presence of two separate molecular clouds. In that case, one should wonder why no absorption line is detected, as in the case of LBV 1806-20, at $\sim 71 \mathrm{~km} \mathrm{~s}^{-1}$ for G10.3-0.1 (Kalberla et al. 1982)? In Fig. 5b, we used the ${ }^{13} \mathrm{CO}(J=1-0)$ of Kim \& Koo (2002) to illustrate the spatial extent of MC 73. We found almost no ${ }^{13} \mathrm{CO}(J=1-0)$ emission in the velocity range 61 to $80 \mathrm{~km} \mathrm{~s}^{-1}$, which is consistent with the non detection of absorption line above $50 \mathrm{~km} \mathrm{~s}^{-1}$ (Kalberla et al. 1982) toward G10.3-0.1, even if it were associated with MC 13A. However, our ${ }^{12} \mathrm{CO}(J=1-0)$ spectrum towards G10.3-0.1 (Fig. 7) indicates a weak contribution of MC 73 along this line sight and suggest that the line of sight of G10.3-0.1 might be close to the edge of MC 73. More sensitive absorption measurement may be useful to further detect absorption line due to MC 73 in front of G10.3-0.1. We note that Kalberla et al. (1980) detected a weak absorption line at $\sim-15 \mathrm{~km} \mathrm{~s}^{-1}$ in front of G10.3-0.1, that is consistent with the velocity of the 3-kpc expanding Arm (Corbel et al. 1997), i.e. the molecular cloud we called MC -16 and the fact that G10.3-0.1 could be associated with MC 13A. We note, however, that a similar but weaker feature, is also present in front of G10.2-0.3 (Kalberla et al. 1980).

In a recent study of absorption lines toward a large number of H II regions, Fish et al. (2003) deduced a scaling law in order to estimate the distance of an $\mathrm{H}$ II region. They state that when there is a "large difference between far and near kinematic distances" - as in our case for both MC 13A and MC 13B, see Table 1 - "high accuracy can be achieved by choosing the kinematic distance closer to $1.84|b|^{-1}$ ". In the case of G10.2-0.3, G10.3-0.1 and G10.6-0.4, this expression would be equal to $5.3,12.3$ and $4.7 \mathrm{kpc}$ respectively with high accuracy ${ }^{2}$. This again will argue for an association of G10.2-0.3 and G10.6-0.4 with MC $13 \mathrm{~B}$ in the $-30 \mathrm{~km} \mathrm{~s}^{-1}$ spiral arm at a distance of $4.5 \pm 0.6 \mathrm{kpc}$ and a location of G10.3-0.1 in MC 13A at a distance of $15.1_{-1.3}^{+1.8} \mathrm{kpc}$. But again, as illustrated in Fig. 3 of Dame et al. (1987), between longitude $5^{\circ}$ and $25^{\circ}$, we want to stress that the lack of absorption at velocities greater than $\sim 50 \mathrm{~km} \mathrm{~s}^{-1}$ is not an argument for the near distance due to the presence of the gas hole (Fig. 6) at low Galactic longitudes (Corbel et al. 1997).

Finally, to close the debate on the structure of W31 (as a location of G10.3-0.1 within the $-30 \mathrm{~km} \mathrm{~s}^{-1}$ spiral arm can not be ruled out at this time), a spectrophotometric study (with optical extinction measurement), similar to what has been performed by Blum et al. (2001) for G10.2-0.3, should also be performed for G10.3-0.1 and G10.6-0.4 in light of our new results. A sketch of the line of sight with the new location of each object introduced in this paper is presented in Fig. 8.

\subsection{The distance to SGR 1806-20}

As we have seen before, G10.0-0.3 is associated with the wind of LBV 1806-20 and not SGR 1806-20 and is not the remnant of a supernova (Gaensler et al. 2001). SGR 1806-20 is

\footnotetext{
${ }^{2}$ Note that this argument cannot be applied to G10.0-0.3, simply because it is not an $\mathrm{H}$ II region.
} 


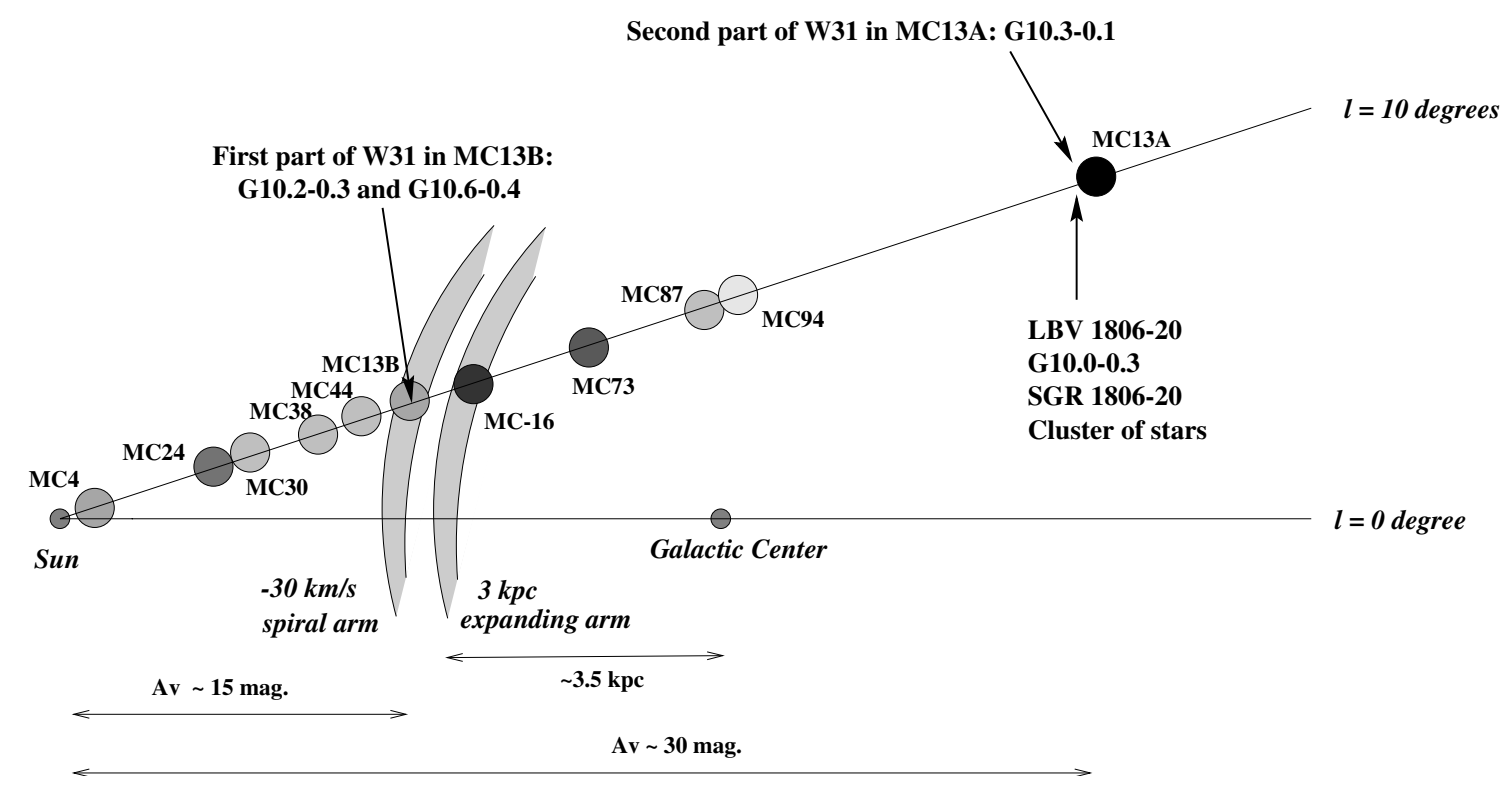

Fig. 8. Schematic diagram of the molecular clouds on the line of sight to W31, LBV 1806-20 and SGR 1806-20.

separated from LBV 1806-20 by an angular distance of $12^{\prime \prime}$ (Hurley et al. 1999; Eikenberry et al. 2001; Kaplan et al. 2002). However, it still lies within the angular extent of the embedded cluster (see Fig. 1 and Fuchs et al. 1999), and its $\mathrm{X}$-ray absorption matches the IR extinction towards the cluster members (Eikenberry et al. 2001), leading to the conclusion that SGR 1806-20 is a cluster member. This lead to the conclusion that all these objects, i.e. SGR 1806-20 and the cluster of stars, have to be located at the distance we have estimated for LBV 1806-20: $15.1_{-1.3}^{+1.8} \mathrm{kpc}$. An interesting consequence of this work is that all these massive stars are still located close to their parental molecular cloud (MC 13A), which is not surprising if we take into account their short lifetimes and the expectation that such massive stars are a kinematically "cold" population. We would like to point out that SGR $1900+14$ is also associated with a cluster of massive stars (Vrba et al. 2000) and that SGR 1627-41 also appears to lie at the edge of a massive Giant Molecular Cloud (Corbel et al. 1999). The only known extragalactic soft gamma repeater (SGR 0526-66), which is associated with the SNR N49, also lies at the edge of a dense molecular cloud (Vancura et al. 1992; Banas et al. 1997). It now also means that all SGRs with precise location (4 out 6) are associated with GMC and/or massive star cluster. All of this probably points to a strong connection between massive stars and formation of SGRs by the way of Giant Molecular Clouds.

\section{Conclusions}

We have presented new millimeter and near-infrared observations of the field of view surroundings the radio nebula G10.0-0.3 (produced by the wind of LBV 1806-20) and the giant H II complex W31. Based on these observations combined with others in the literature, we reach the following conclusions:

- Based on $\mathrm{NH}_{3}$ absorption from $\mathrm{MC} 73$ and the velocity of LBV 1806-20, we unambiguously constrain the distance to G10.0-0.3 and LBV 1806-20 to be in the range of $13.2-21.5 \mathrm{kpc}$.

- Combining this constraint with CO observations of molecular clouds along the line of sight, we further refine the distance measurement to G10.0-0.3 and LBV 1806-20 to be $15.1_{-1.3}^{+1.8} \mathrm{kpc}$.

- This distance estimate is confirmed by the consistency between the measured extinction towards LBV 1806-20 and the extinction from the molecular clouds along the line of sight inferred from their CO spectra.

- Based on their distinct extinctions and the newly-resolved parental molecular clouds (MC 13A and MC 13B), we conclude that the stellar cluster in G10.2-0.3 lies in the foreground to the cluster containing LBV 1806-20. This shows that W31 consists of at least 2 discrete components along the line of sight.

- We suggest that G10.2-0.3 and G10.6-0.4 are located on the $-30 \mathrm{~km} \mathrm{~s}^{-1}$ spiral arm at a distance from the Sun of $4.5 \pm 0.6 \mathrm{kpc}$.

- We also suggest that G10.3-0.1 may be associated with a massive molecular cloud at the same distance as LBV 1806-20 (15.1 $\left.1_{-1.3}^{+1.8} \mathrm{kpc}\right)$.

- We confirm that SGR 1806-20 is located at a distance from the Sun of $15.1_{-1.3}^{+1.8} \mathrm{kpc}$ and that it is associated with a very massive molecular cloud. All SGRs with precise location are associated with a site of massive star formation.

Acknowledgements. The authors would like to thank Tom Dame and Kee-Tae Kim for useful discussions and for critical review of this manuscript. We also wish to thank Claude Chapuis for conducting the 1998 SEST observations and stimulating discussion and Bill Mahoney for carrying out the DSN observations. We thank R. Blum and the CTIO staff for their help in acquiring the IR spectra. We gratefully acknowledge Kee-Tae Kim and Bon-Chul Koo for sharing their CO data of W31, as well as Yael Fuchs for her help with Fig. 1 and Tom Dame for his help with Fig. 6. We also thank Aage Sandqvist for providing information on the $-30 \mathrm{~km} \mathrm{~s}^{-1}$ spiral arm. SSE is supported in part by an NSF CAREER award (AST-9983830). 


\section{References}

Banas, K. R., Hughes, J. P., Bronfman, L., \& Nyman, L.-A. 1997, ApJ, 480, 607

Bania, T. M. 1980, ApJ, 242, 95

Blitz, L., \& Spergel, D. N. 1991, ApJ, 379, 631

Blum, R. D., Damineli, A., \& Conti, P. S. 2001, ApJ, 121, 3149

Chapuis, C., \& Corbel, S. 2004, A\&A, 414, 659

Corbel, S., Wallyn, P., Dame, T. M., et al. 1997, ApJ, 478, 624

Corbel, S., Chapuis, C., Dame, T. M., \& Durouchoux, P. 1999, ApJ, 526, L29

Combes, F. 1991, ARA\&A, 29, 195

Dame, T. M., Ungerechts, H., Cohen, R. S., et al. 1987, ApJ, 322, 706

Dame, T. M., Hartmann, D., \& Thaddeus, P. 2001, ApJ, 547, 792

Depoy, D., Atwood, B., Byard, P. L., Frogel, J., \& O’Brien, T. P. 1993 , SPIE, 1946, 667

Eikenberry, S. S., Garske, M. A., Hu, D., et al. 2001, ApJ, 563, L133

Eikenberry, S. S., Matthews, K., Garske, M. A., et al., ApJ, accepted

Fich, M., Blitz, L., \& Stark, A. A. 1989, ApJ, 342, 272

Fish, V. L., Reid, M. J., Wilner, D. J., \& Churchwell, E. 2003, ApJ, 587,701

Fuchs, Y., Mirabel, F., Chaty, S., et al. 1999, A\&A, 350, 891

Gaensler, B. M., Slane, P. O., Gotthelf, E. V., \& Vasisht, G. 2001, ApJ, 559,963

Ghosh, S. K., Iyengar, K. V. K., Rengarajan, T. N., et al. 1989, ApJ, 347,338

Greaves, J. S., \& Williams, P. G. 1994, A\&A, 290, 259

Greisen, E. W., \& Lockman, F. J. 1979, ApJ, 228, 740

Hurley, K., Kouveliotou, C., Cline, T., et al. 1999, ApJ, 523, L37

Kalberla, P. M. W., Goss, W. M., \& Wilson, T. L. 1982, A\&A, 106, 167
Kaplan, D. L., Fox, D. W., Kulkarni, S. R., et al. 2002, ApJ, 564, 935

Kerr, F. J., \& Vallak, R. 1967, Austr. J. Phys., Astrophys. Suppl., 3, 3

Kim, K.-T., \& Koo, B.-C. 2001, ApJ, 549, 979

Kim, K.-T., \& Koo, B.-C. 2002, ApJ, 575, 327

Kouveliotou, C., Dieters, S., Strohmayer, T., et al. 1998, Nature, 393, 235

Kulkarni, S. R., \& Frail, D. A. 1993, Nature, 365, 33

Kulkarni, S. R., Frail, D. A., Kassim, N. E., Murakami, T., \& Vasisht, G. 1994, Nature, 368, 129

Linke, R. A., Stark, A. A., \& Frerking, M. A. 1981, ApJ, 243, 147

Lavine, J., Eikenberry, S. S., \& Smith, J. D., in preparation

Liszt, H. S., Burton, W. B., Sanders, R. H., \& Scoville, N. Z. 1977, ApJ, 213, 38

Menon, T. K., \& Ciotti, J. E. 1970, Nature, 227, 579

Mereghetti, S., Cremonesi, D., Feroci, M., \& Tavani, M. 2000, A\&A, 361,240

Rieke, G. H., \& Lebofsky, M. J. 1985, ApJ, 288, 618

Sandqvist, A., Bergman, P., Black, J. H., et al. 2003, A\&A, 402, L63

Shaver, P. A., \& Goss, W. M. 1970, Austr. J. Phys., Astrophys. Suppl., 14,77

van Kerkwijk, M. H., Kulkarni, S. R., Matthews, K., \& Neugebauer, G. 1995, ApJ, 444, L33

Vancura, O., Blair, W. P., Long, K. S., \& Raymond, J. C. 1992, ApJ, 394, 158

Vasisht, G., Frail, D. A., \& Kulkarni, S. R. 1995, ApJ, 440, L65

Vrba, F. J., Henden, A. A., Luginbuhl, C. B., et al. 2000, ApJ, 533, $\mathrm{L} 17$

Wilson, T. L. 1974, A\&A, 31, 83 